\title{
Mature high-affinity immune responses to (pro)insulin anticipate the autoimmune cascade that leads to type 1 diabetes
}

\author{
Peter Achenbach, ${ }^{1}$ Kerstin Koczwara, ${ }^{1}$ Annette Knopff, ${ }^{1}$ Heike Naserke, ${ }^{1}$ \\ Anette-G. Ziegler, ${ }^{1}$ and Ezio Bonifacio, ${ }^{1,2}$ \\ ${ }^{1}$ Institut für Diabetesforschung, Munich, Germany. ${ }^{2}$ sstituto Scientifico San Raffaele, Milan, Italy.
}

\begin{abstract}
Children at risk for type 1 diabetes can develop early insulin autoantibodies (IAAs). Many, but not all, of these children subsequently develop multiple islet autoantibodies and diabetes. To determine whether disease progression is reflected by autoantibody maturity, IAA affinity was measured by competitive radiobinding assay in first and subsequent IAA-positive samples from children followed from birth in the BABYDIAB cohort. IAA affinity in first positive samples ranged from less than $10^{6} 1 / \mathrm{mol}$ to more than $10^{11} 1 / \mathrm{mol}$. High affinity was associated with $H L A D R B 1 * 04$, young age of IAA appearance, and subsequent progression to multiple islet autoantibodies or type 1 diabetes. IAA affinity in multiple antibody-positive children was on average 100-fold higher than in children who remained single IAA positive or became autoantibody negative. All high-affinity IAAs required conservation of human insulin A chain residues 8-13 and were reactive with proinsulin. In contrast, most lower-affinity IAAs were dependent on $\mathrm{COOH}$-terminal B chain residues and did not bind proinsulin. These data are consistent with the concept that type 1 diabetes is associated with sustained early exposure to (pro)insulin in the context of HLA DR4 and show that high-affinity proinsulin-reactive IAAs identify children with the highest diabetes risk.
\end{abstract}

\section{Introduction}

During the natural history of childhood diabetes, insulin autoantibodies (IAAs) are often the first autoantibody detected early in infancy (1-5). Many, but not all, IAA-positive children also develop autoantibodies to other $\beta$ cell antigens such as glutamic acid decarboxylase (GAD) and the protein tyrosine phosphataselike proteins IA-2 and IA-2 $\beta(2-4)$. Children who also develop these antibodies usually progress to clinical type 1 diabetes mellitus (T1DM), whereas children who remain positive only for IAAs rarely develop T1DM (2). Development of multiple islet autoantibodies is, therefore, an important step in the pathogenesis of the disease.

There is no marker that distinguishes the IAA-positive children who will eventually become multiple antibody-positive. In a typical antibody response, exposure to antigen in the presence of $\mathrm{B}$ cell growth factors results in B lymphocyte expansion and IgM antibody production. Sustained or repeated antigen exposure results in a switch from IgM to IgG production, and subsequent exhaustion of antigen leads to the selection of clones that produce high-affinity antibodies to the antigen $(6,7)$. Affinity could, therefore, reflect a stage of antigen encounter and, in the case of IAAs, may be useful in staging the preclinical phase of T1DM. In order to address whether autoantibody affinity matures during preclinical diabetes and whether it predicts progression to multiple islet autoantibodies, IAA affinity was measured in IAA-positive children from the prospectively followed BABYDIAB cohort (8). The findings in these children who are followed during infan$\mathrm{cy}$ indicate that IAA affinity is fixed relatively early in the autoim-

Nonstandard abbreviations used: glutamic acid decarboxylase (GAD); insulin autoantibody (IAA); interquartile range (IQR); type 1 diabetes mellitus (T1DM). Conflict of interest: The authors have declared that no conflict of interest exists.

Citation for this article: J. Clin. Invest. 114:589-597 (2004).

doi:10.1172/JCI200421307. mune response, that it distinguishes IAAs with different epitope reactivity, and that it identifies IAA-positive children who will progress to multiple autoantibodies.

\section{Results}

IAA binding characteristics are consistent with a 1-site binding model and therefore relatively homogeneous within samples. IAAs are measured by radiobinding assay using $\left[{ }^{125} \mathrm{I}\right]$ insulin labeled at tyrosine at position 14 of the A chain. To determine whether iodine labeling at this position affected the binding of autoantibodies and therefore IAA affinity measurements, [ $\left.{ }^{125} \mathrm{I}\right]$ insulin labeled at 1 of 3 different tyrosine residues of the insulin $\mathrm{A}$ chain $\left(\operatorname{Tyr}^{14} \mathrm{~A}, \operatorname{Ty}^{19} \mathrm{~A}\right)$ or $\mathrm{B}$ chain $\left(\operatorname{Tyr}^{16} \mathrm{~B}\right)$ were used to measure affinity in an IAA-positive relative. Although the nonspecific binding was increased when $\mathrm{Tyr}^{16} \mathrm{~B}$ insulin was used as label, binding curves and $\mathrm{IC}_{50}$ and $K_{\mathrm{d}}$ values were similar with the $\left[{ }^{125} \mathrm{I}\right]$ insulin labeled at residues $\operatorname{Tyr}^{14} \mathrm{~A}, \operatorname{Tyr}^{19} \mathrm{~A}$, or ${ }^{T y r}{ }^{16} \mathrm{~B}$ (Figure $1 \mathrm{~A}$ ), indicating that $\operatorname{Tyr}^{14} \mathrm{~A}$ iodine labeling is unlikely to interfere with the binding of IAAs.

The IAA binding curve observed in the IAA-positive sample was consistent with a 1-site binding model (Figure 1, A and B) and IAAs of high affinity $\left(1.7 \times 10^{11} \mathrm{l} / \mathrm{mol}\right)$. In order to determine whether mixed IAA populations of discrete affinities could be identified by the experimental system, mixing experiments were performed by spiking of the high-affinity IAA-positive serum with serum containing low-affinity $\left(2 \times 10^{5} \mathrm{l} / \mathrm{mol}\right)$ or moderate-affinity $\left(6.3 \times 10^{7} \mathrm{l} /\right.$ mol) IAAs (Figure 1C). The competitive binding curves of the mixed sera were consistent with a 2-site binding model, and the calculated IAA affinities for each of the IAAs were similar to those determined in the original samples (high-low mix, $1.6 \times 10^{11} \mathrm{l} / \mathrm{mol}$ and $2.8 \times 10^{5}$ $1 / \mathrm{mol}$; high-moderate mix, $1.2 \times 10^{11} \mathrm{l} / \mathrm{mol}$ and $6.2 \times 10^{7} \mathrm{l} / \mathrm{mol}$ ).

IAA competitive binding curves to $\operatorname{Tyr}^{14} \mathrm{~A}\left[{ }^{125} \mathrm{I}\right]$ insulin were consistent with a 1-site model in the first IAA-positive sample from all but 1 of the 56 children tested, suggesting that IAAs were of 

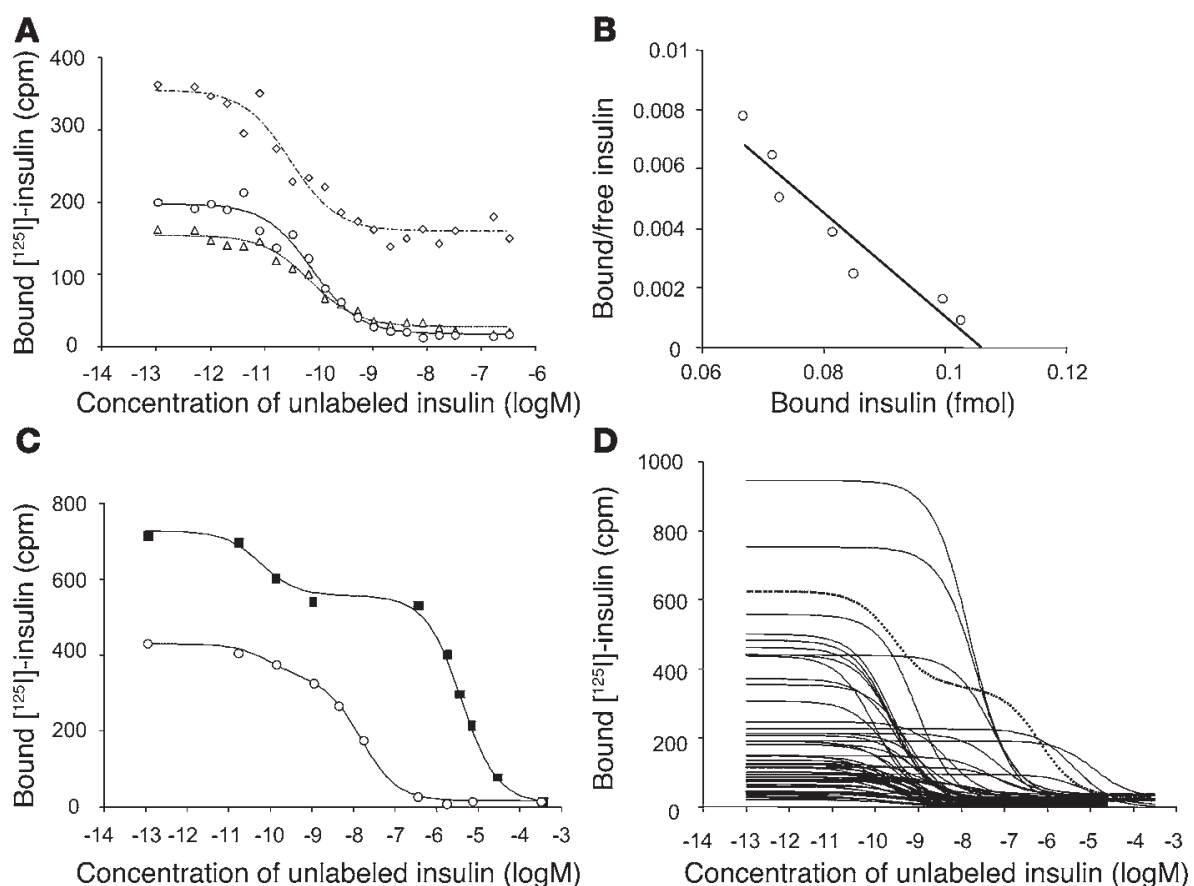

Figure 1

Competitive insulin binding curves of IAAs. (A) Competition of an IAA-positive serum against [125I] insulin labeled at $\mathrm{Ty}^{14} \mathrm{~A}$ (circles), $\mathrm{Ty}^{19} \mathrm{~A}$ (triangles), and $\mathrm{Ty}^{16} \mathrm{~B}$ (diamonds) with increasing concentrations of unlabeled human insulin. Binding curves were similar with the [125I] insulin labeled at residues $\operatorname{Tyr}^{14} \mathrm{~A}$, $\operatorname{Tyr}^{19} \mathrm{~A}$, or $\mathrm{Tyr}^{16} \mathrm{~B}$, and calculated IAA affinities did not significantly differ among $\mathrm{Tyr}^{14} \mathrm{~A}$ $\left[{ }^{125} \mathrm{l}\right]$ insulin $\left(2.1 \times 10^{11} \mathrm{l} / \mathrm{mol}\right)$, Tyr ${ }^{19} \mathrm{~A}\left[{ }^{125} \mathrm{l}\right]$ insulin $\left(2.3 \times 10^{11} \mathrm{l} / \mathrm{mol}\right)$, and $\mathrm{Tyr}^{16} \mathrm{~B}\left[{ }^{125} \mathrm{l}\right]$ insulin $\left(5.7 \times 10^{11}\right.$ $\mathrm{I} / \mathrm{mol}$ ). Insulin labeled at position $\mathrm{Ty}^{16} \mathrm{~B}$ was associated with considerably higher nonspecific binding than insulin labeled at $\mathrm{Tyr}^{14} \mathrm{~A}$ or $\mathrm{Ty}^{19} \mathrm{~A}$. (B) Scatchard analysis performed for the competition curve obtained against $\mathrm{Tyr}^{14} \mathrm{~A}$ radiolabeled insulin. (C) Binding curves of a serum mix containing a serum with high-affinity IAAs $\left(1.7 \times 10^{11} \mathrm{I} / \mathrm{mol}\right)$ and a serum with low-affinity IAAs $\left(2 \times 10^{5} \mathrm{I} / \mathrm{mol}\right)$ (squares) and a serum mix containing a serum with high-affinity IAAs $\left(1.7 \times 10^{11} \mathrm{I} / \mathrm{mol}\right)$ and a serum with moderate-affinity IAAs $\left(6.3 \times 10^{7} \mathrm{l} / \mathrm{mol}\right)$ (circles). Both curves fit a 2-site binding model. (D) Binding curves obtained for the first IAA-positive serum from 56 children in the BABYDIAB study. IAA binding in 1 serum conforms to a 2-site binding model (dotted line), whereas the remaining sera conform to a 1-site binding model. Curves that are shifted to the right indicate lower-affinity IAAs.

relatively homogeneous affinity within each sample (Figure 1D). The IAA binding curve in 1 child aged 9 months was consistent with a 2 -site binding model.

IAA affinity varies between children. IAA affinity in the first positive sample from the BABYDIAB children varied substantially between children and ranged from less than $10^{6} 1 / \mathrm{mol}$ to more than $10^{11} \mathrm{l} / \mathrm{mol}$ (Figure 2 ). Affinity was not correlated with IAA titer $(r=-0.016 ; P=0.91)$ but was associated with $H L A D R B 1 * 04$ (median affinity, $5.4 \times 10^{9} \mathrm{l} / \mathrm{mol}$ in $H L A D R B 1 * 04$ children vs. $9.3 \times 10^{8} \mathrm{l} / \mathrm{mol}$ in non-DRB1*04 children; $\left.P=0.002\right)$ and the age of first IAA detection $(P=0.003$, Kruskal-Wallis $H$ test). IAA affinity was greater than $10^{9} \mathrm{l} / \mathrm{mol}$ in all 16 children who had IAAs at 9 months (median affinity, $6.8 \times 10^{9} \mathrm{l} / \mathrm{mol}$ ). Only 4 of these 16 children were positive at birth, so the high-affinity IAAs were not due to residual maternal insulin antibodies. The majority $(69 \%)$ of children in whom IAAs were first detected at age 2 years also had high-affinity IAAs (median affinity, $3.6 \times 10^{9}$ $1 / \mathrm{mol}$ ). In contrast, only 3 of 14 children in whom IAAs were first detected at age 5 or 8 years had affinities above $10^{9} \mathrm{l} / \mathrm{mol}$ (median affinity, $2.7 \times 10^{8} \mathrm{l} / \mathrm{mol} ; P=0.004 \mathrm{vs}$. IAA affinity in children who developed IAAs at age 9 months or 2 years).
IAA affinity is high in children who develop multiple islet autoantibodies. IAA affinity was analyzed with respect to progression to multiple islet autoantibodies and to diabetes (Figure 3A). IAA affinity in the first IAA-positive sample was significantly higher in the 38 children who developed multiple islet autoantibodies (median IAA affinity, $5.4 \times 10^{9} \mathrm{l} / \mathrm{mol}$; interquartile range [IQR], $2.7 \times 10^{9}$ to $1.3 \times 10^{10} \mathrm{l} / \mathrm{mol}$ ) than in the 18 children who did not develop multiple antibodies (median, $5.2 \times 10^{7} \mathrm{l} / \mathrm{mol}$; IQR, $1.2 \times 10^{7}$ to $\left.7.0 \times 10^{8} \mathrm{l} / \mathrm{mol} ; P<0.0001\right)$. Thirty-six of the 38 children who developed multiple islet autoantibodies and all 20 children who developed T1DM had IAA affinities greater than $10^{9} \mathrm{l} / \mathrm{mol}$, compared with only 2 of 18 of the children who did not progress to multiple islet autoantibodies, including none of 5 who later became IAA negative (transient IAA). IAA affinity in a second group of IAA-positive relatives (Munich family study) was also significantly higher in relatives who had or developed multiple islet autoantibodies (median affinity, $6.9 \times 10^{9} \mathrm{l} / \mathrm{mol}$ ) than in relatives who did not progress to multiple islet autoantibodies (median affinity, $\left.8.1 \times 10^{5} \mathrm{l} / \mathrm{mol} ; P=0.002\right)$. Progression to multiple antibodies in both cohorts was not related to IAA titer (Figure 3B).

In comparison, affinity of insulin antibodies in patients after treatment with subcutaneous insulin was high (median affinity, $2.0 \times 10^{9} \mathrm{l} / \mathrm{mol}$ ) and remarkably consistent between patients (IQR, $1.7 \times 10^{9}$ to $2.2 \times 10^{9} \mathrm{l} / \mathrm{mol}$; Figure $3 \mathrm{~A})$. In contrast, IAA affinities in 2 sera from blood donors found to be IAA positive in the Diabetes Autoantibody Standardization Program (9) were low (sample M66290, $2.0 \times 10^{5} \mathrm{l} / \mathrm{mol}$; sample N05151, $9.2 \times 10^{6} \mathrm{l} / \mathrm{mol}$ ).

High IAA affinity identifies individuals who later progress to multiple islet autoantibodies. Thirty-three of the IAA-positive BABYDIAB children tested did not have other islet autoantibodies in their first IAA-positive sample. In order to determine whether measuring IAA affinity would be useful for distinguishing IAA-positive relatives who would develop multiple islet autoantibodies on follow-up, time-to-event analyses were performed in these 33 children (Figure 4). Progression to multiple islet autoantibodies was $91 \%$ within 4 years of follow-up in the 16 children with high-affinity IAAs $\left(>10^{9} \mathrm{l} / \mathrm{mol}\right)$ and was significantly more frequent than in the 17 children with low-affinity IAAs $\left(<10^{9} \mathrm{l} / \mathrm{mol}\right.$; $P=0.0004$; Figure 4A). One child with low-affinity IAAs developed multiple islet autoantibodies. Progression to multiple islet autoantibodies in this child was accompanied by a marked increase in IAA affinity. Risk to develop diabetes in the children with high-affinity IAAs was $50 \%$ within 6 years $(95 \%$ confidence interval; 32.1-67.9), whereas none of the children with low-affinity IAAs has developed diabetes $(P=0.02$; Figure 4B). 


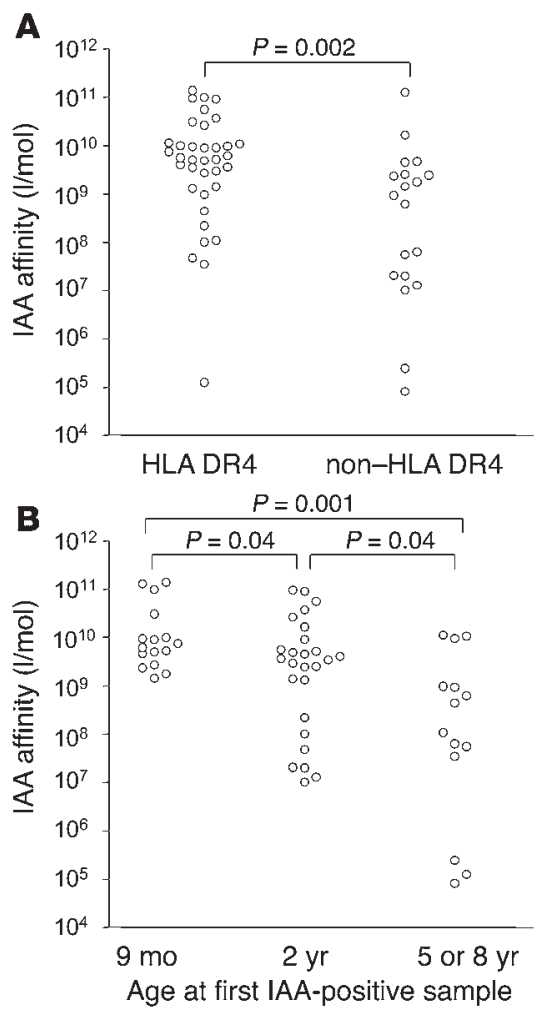

IAA affinity is relatively stable during follow-up. The findings indicated that a high-affinity IAA response could occur early in the natural history of T1DM, and that this was predictive of who would develop multiple islet autoantibodies and diabetes. In order to determine whether the lower-affinity responses "matured" and became of higher affinity later in childhood, IAA affinity was determined in 92 follow-up sera from 31 children (Figure 5A). Changes that were greater than $1 \log$ were observed in 4 of these 31 children. IAA affinity increased in follow-up in only 1 of the 11 children with initial IAA affinity less than $10^{9} \mathrm{l} / \mathrm{mol}$. This child was remarkable in that IAAs were positive at age 2 years with an affinity of $10^{8} \mathrm{l} / \mathrm{mol}$, became negative at age 2.7 years, and returned to positive with increased affinity $\left(1.4 \times 10^{11} \mathrm{l} / \mathrm{mol}\right)$ together with GAD antibodies at age 5 years. IAA affinity in a second child increased from $2.7 \times 10^{9} \mathrm{l} / \mathrm{mol}$ to $7.6 \times 10^{10}$ $1 /$ mol. Two children had decreased IAA affinity on follow-up.

IAA binding curves in follow-up samples from the child with concomitant high- and low-affinity IAAs were informative with

\section{Figure 3}

Relationship between IAA affinity, multiple autoantibodies, and diabetes. (A) IAA affinity $(\mathrm{I} / \mathrm{mol})$ in the first IAA-positive sample from 56 children in the BABYDIAB study, in 16 IAA-positive relatives from the Munich family study, and in 11 insulin-treated patients with T1DM. Subjects in the BABYDIAB and Munich family studies are classified as having developed GAD antibodies, IA-2 antibodies, or cytoplasmic islet cell autoantibodies (multiple Ab's) or not having developed these antibodies (IAAs only). (B) Relationship between IAA affinity (ordinate scale) and IAA titer (abscissa) for the 72 BABYDIAB and Munich family study sera. In $\mathbf{A}$ and $\mathbf{B}$, subjects are identified as having developed multiple islet autoantibodies (circles), not having developed multiple islet autoantibodies (crosses), or having transient IAAs (triangles), and as having developed diabetes (filled symbols) or not (open symbols).

\section{Figure 2}

Relationship between IAA affinity and the age of IAA appearance or HLA phenotype. (A) IAA affinity of the first IAA-positive sample in children who had the HLA DRB1*04/DQB1*0302 haplotype (HLA DR4) compared with those who did not have this haplotype (non-HLA DR4). (B) IAA affinity of the first IAA-positive sample in BABYDIAB children who developed IAAs at age 9 months or 2 years or at 5 years or older.

respect to affinity maturation (Figure 5B). Samples were available at birth and at approximately 3 -month intervals from ages 6 months to 21 months. Insulin antibodies at birth and at 6 months were high affinity $\left(7 \times 10^{9} \mathrm{l} / \mathrm{mol}\right)$ without a low-affinity component, consistent with the presence of maternally acquired antibodies to injected insulin. At age 9 months, insulin binding increased markedly. A high-affinity component was present with titer greater than that observed in the 6-month sample, indicating that this included de novo production of IAAs in the child. A predominant low-affinity IAA was also present. Subsequent samples at ages $12,15,18$, and 21 months had decreasing amounts of both the high- and the low-affinity IAAs until the low-affinity component became undetectable at 18 months. None of the samples from this child contained IgM IAAs (data not shown). This child developed diabetes at age 2.8 years.

Lower-affinity IAAs show a less mature isotype and a restricted $\operatorname{IgG}$ subclass distribution. IAA IgG subclasses were measured in the first IAA-positive samples from 44 of the BABYDIAB children. These included 31 with IAA affinity greater than $10^{9} \mathrm{l} / \mathrm{mol}$ (30 of whom developed multiple islet autoantibodies) and 13 with IAA affinity less than $10^{\circ} \mathrm{l} / \mathrm{mol}$. All 31 with high-affinity IAAs had IgG1 IAAs, and 19 of these also had IgG2, IgG3, or IgG4 IAAs. In contrast,
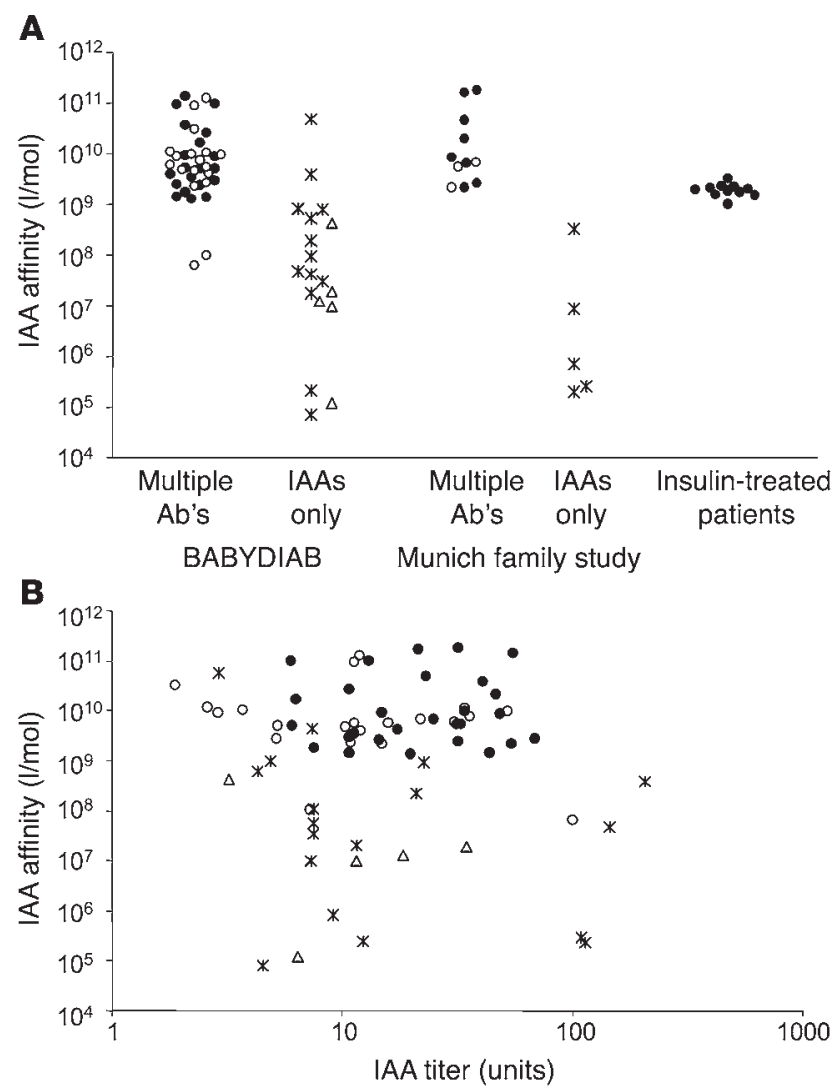
A

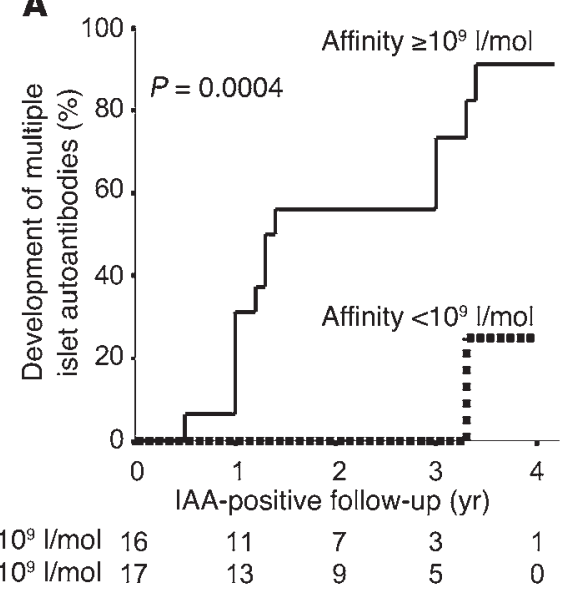

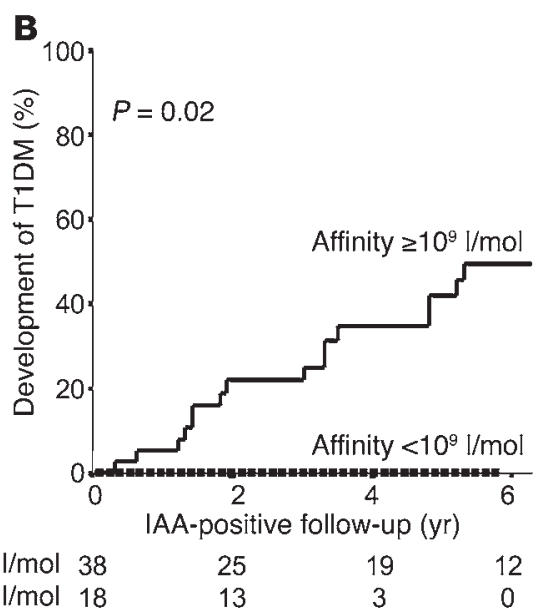

\section{Figure 4}

Progression to multiple autoantibodies (A) and diabetes (B) with respect to IAA affinity. Life table analysis of the development of multiple islet autoantibodies was done in 33 BABYDIAB children who were IAA positive without other autoantibodies in their first positive sample. Life table analysis of the development of diabetes was performed for all 56 BABYDIAB children included in the study. Children are categorized as having IAA affinity greater than $10^{9} \mathrm{l} / \mathrm{mol}$ (solid line) or less than $10^{9}$ $\mathrm{I} / \mathrm{mol}$ (dotted line). Multiple antibodies and diabetes developed more frequently in children with IAA affinity greater than $10^{9} \mathrm{l} / \mathrm{mol}(P=0.0004$ and $P=0.02$, respectively).
11 of the 13 children with low-affinity IAAs had IAAs consisting of only $1 \mathrm{IgG}$ subclass $(P=0.008$ vs. children with high-affinity IAAs), including 1 child with IgG2 IAAs, 1 with IgG3 IAAs, and 1 with IgG4 IAAs (data not shown). The restricted IgG subclass in the lower-affinity IAA samples was independent of IAA titer, which was similar in high- and low-affinity samples.

$\operatorname{IgM}$ antibodies are of lower affinity than IgG antibodies. Two children with high-titer low-affinity IAAs $\left(<10^{6} \mathrm{l} / \mathrm{mol}\right)$ had high titers of IgM IAAs, and their IAA binding was abolished when the reaction was performed at room temperature, indicating that these were cold-reactive IgM antibodies to insulin. All the other sera tested had predominantly IgG IAAs, did not have a large IgM component of IAAs, and were reactive both at $4{ }^{\circ} \mathrm{C}$ and at room temperature (data not shown).

Affinity identifies IAAs with distinct insulin binding characteristics. The wide range of IAA affinity found between subjects suggested that there were substantial differences in the IAA-insulin interaction. We therefore examined binding to alternatively labeled insulin and insulin from different species or insulin analogs. Binding to Tyr ${ }^{19} \mathrm{~A}\left[{ }^{125} \mathrm{I}\right]$ insulin was markedly reduced relative to binding to $\operatorname{Tyr}^{14} \mathrm{~A}\left[{ }^{125} \mathrm{I}\right]$ insulin in some patients (Figure 6). Binding to $\mathrm{Tyr}^{19} \mathrm{~A}$ $\left.{ }^{[25} \mathrm{I}\right]$ insulin was significantly correlated to IAA affinity $(r=0.57$; $P=0.001)$, and IAAs of very low affinity did not bind to $\mathrm{Tyr}^{19} \mathrm{~A}\left[{ }^{125} \mathrm{I}\right]$ insulin, even when titers against $\operatorname{Tyr}^{14} \mathrm{~A}\left[{ }^{125} \mathrm{I}\right]$ insulin were high.

The affinity-related interference with IAA binding caused by labeling at residue A19 could be explained by steric hindrance of sufficient magnitude to reduce binding of the lower-affinity IAAs, or by IAA epitope differences. In order to determine whether the lower-affinity IAAs were directed against distinct

\section{Figure 5}

IAA affinity during follow-up. (A) IAA affinity over time (age) for 92 follow-up samples from 31 subjects. Samples are identified as multiple islet autoantibody positive (circles) or IAA positive only (crosses). Samples from individual subjects are connected by lines. IAA affinity increased by more than 1 log in only 2 subjects (thick broken line) and decreased by more than 1 log in 2 subjects (dotted lines). (B) IAA competitive binding curves for consecutive samples from birth in an IAA-positive BABYDIAB child whose sample at age 9 months had binding characteristics consistent with a 2 -site binding model. Binding on the ordinate scale is shown as binding $(B)$ relative to maximal binding in the absence of cold insulin (B0). The inset documents IAA titer and affinity at each follow-up visit. epitopes, we performed competition studies using modified insulin in 65 IAA-positive subjects from the BABYDIAB $(n=54)$ and Munich family study $(n=11)$ cohorts (Figure 7 , $A$ and $B$, and Table 1$)$. The majority of subjects $(n=46)$ had IAAs that bound equally well to human, porcine, and human B28lysB29pro insulin, bound less to sheep A8his insulin and human A13trpB28lysB29pro insulin, and did not bind fish insulin. This pattern corresponded to binding that required conservation of the human sequence within $\mathrm{A}$ chain residues 8-10 and 13, but not B chain residues 28-30 (A8-10/13-dependent binding). A second group of subjects $(n=6)$ had IAAs that bound equally well to all insulins and insulin analogs except fish insulin, which indicated that they were unaffected by changes at A chain residues 8-10 or 13 or B chain residues $28-30$ (A8-10/13/B28-30-independent binding). A third group of
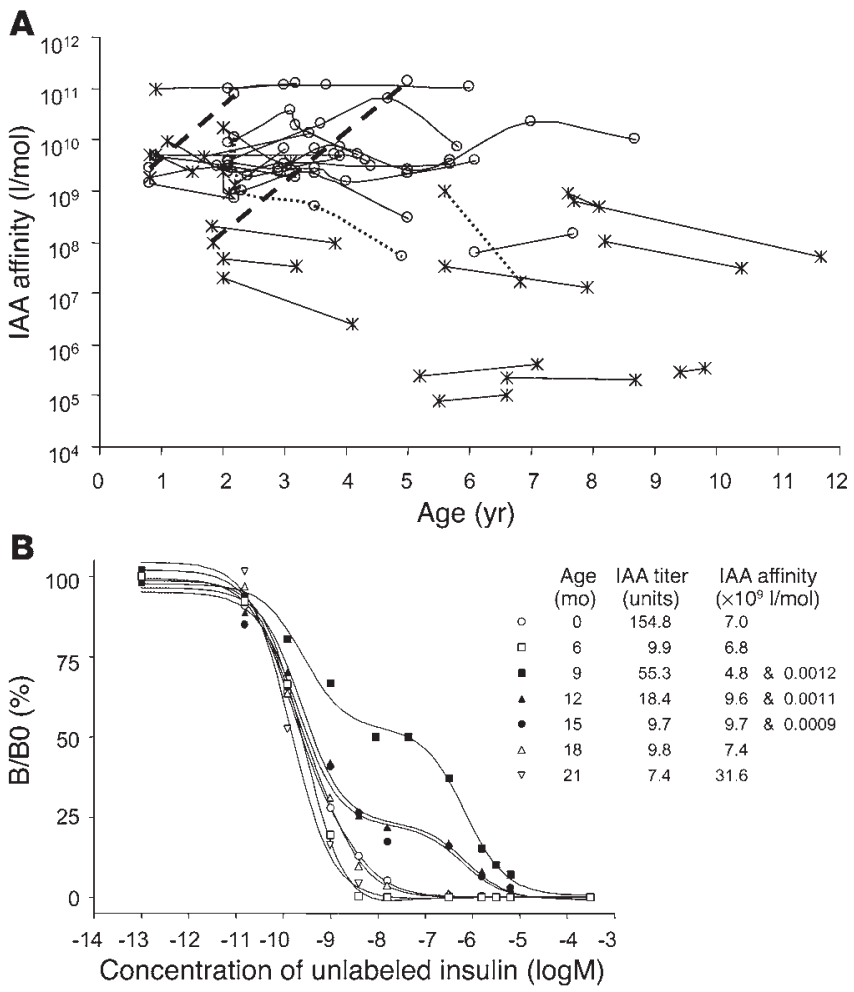


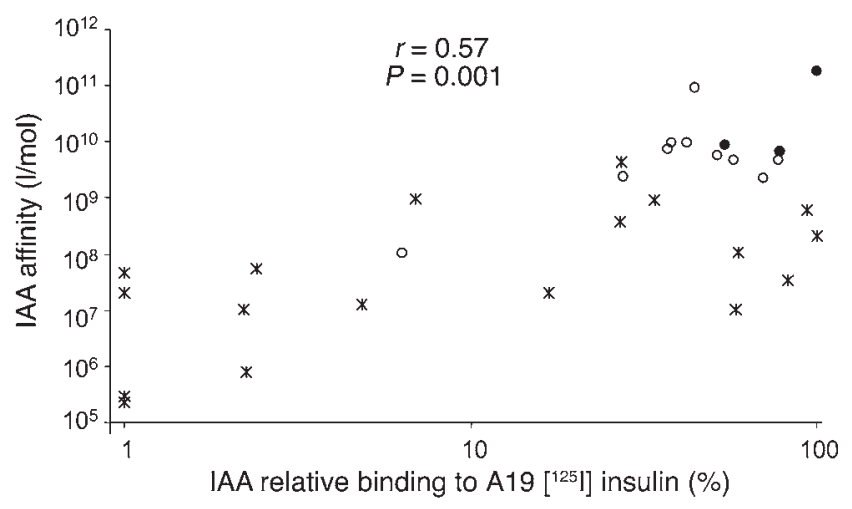

subjects $(n=13)$ had IAAs that were affected by changes to insulin B chain residues 28,29 , or 30 (B28-30-dependent binding). These included 3 subjects with IAAs that did not bind to porcine

\section{Figure 6}

Relationship between IAA affinity and relative binding to $T y r^{19} \mathrm{~A}\left[{ }^{125} \mathrm{I}\right]$ insulin. Percent binding to $\operatorname{Tyr}^{19} \mathrm{~A}\left[{ }^{125} \mathrm{I}\right]$ insulin relative to binding to $\operatorname{Tyr}^{14} \mathrm{~A}\left[{ }^{125}\right.$ ] insulin (abscissa) is shown in relation to IAA affinities (ordinate axis) for individual sera. Children who had or developed multiple islet autoantibodies are indicated by circles and those who did not develop multiple islet antibodies by crosses. Filled symbols represent children who developed diabetes.

insulin, 3 with IAAs that did not bind to human B28lysB29pro insulin (but bound well to fish insulin), and 7 with IAAs that bound neither human B28lysB29pro nor porcine insulin.

All 44 subjects with high-affinity IAAs had the A8-10/13-dependent binding pattern. In contrast, of the 21 subjects with lower IAA affinity, 13 had IAAs with a B28-30-dependent binding pattern $(P<0.0001$ vs. high-affinity IAAs), 6 had IAAs with A8-10/13/ B28-30-independent binding $(P=0.0007)$, and only 2 had IAAs with the A8-10/13-dependent binding pattern $(P<0.0001)$.

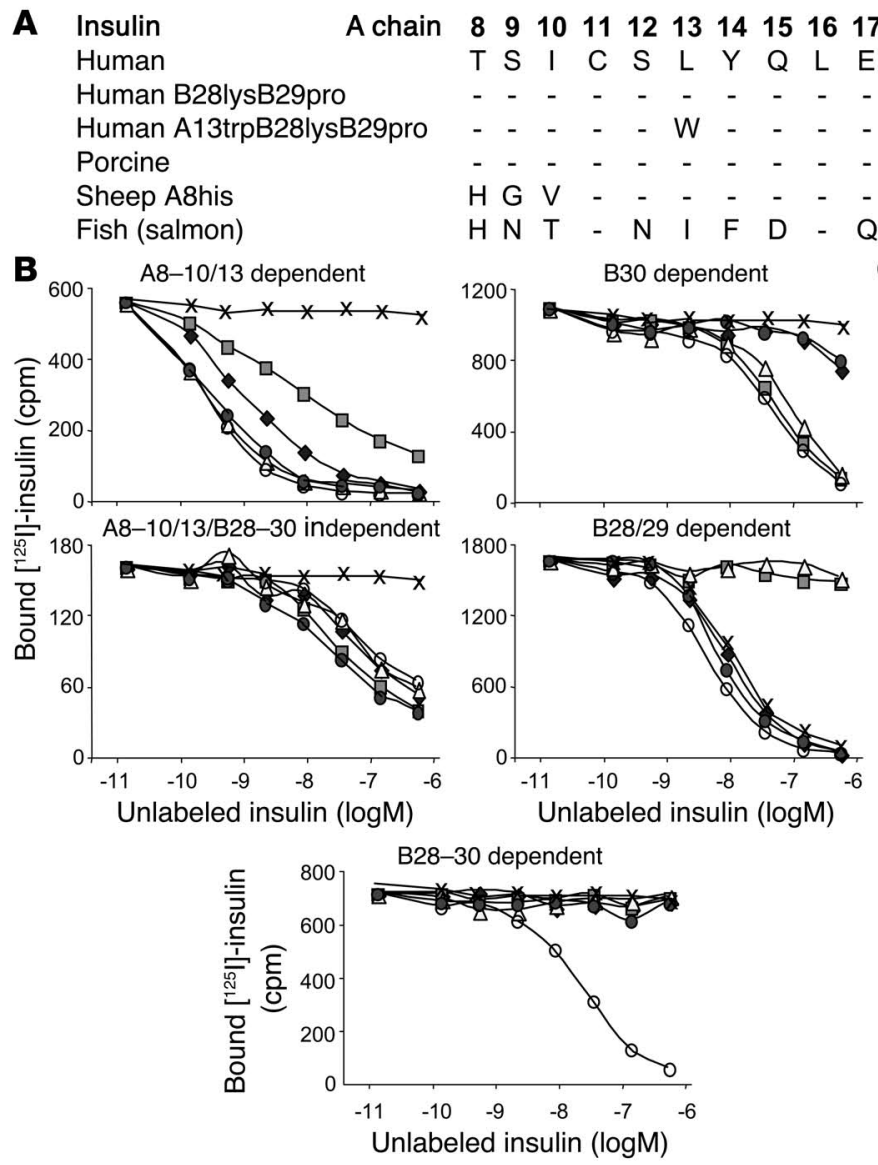

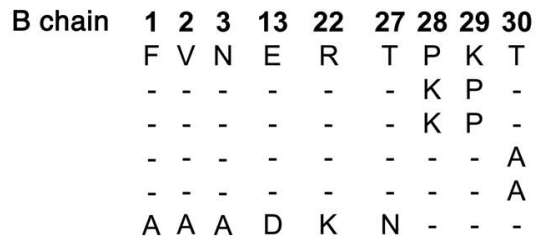
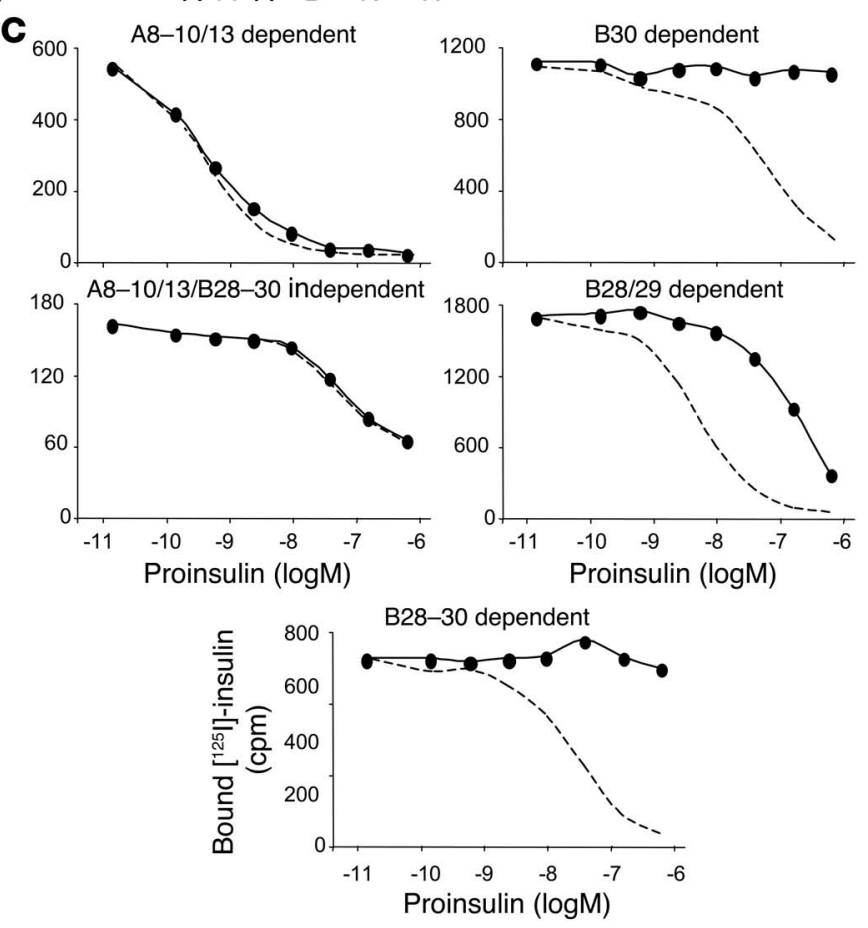

Figure 7

Epitope analysis of IAA. (A) Differences in amino acid sequences in the A and B chains of the insulin molecules used for competition studies of IAA binding. (B) Competitive inhibition of IAA binding to Tyr ${ }^{14} A$ [ ${ }^{125}$ I] human insulin using human insulin (open circles), human B28lysB29pro insulin (open triangles), human A13trpB28lysB29pro insulin (shaded squares), porcine insulin (shaded circles), sheep A8his insulin (filled diamonds), and fish insulin (crosses). Five patterns were discernible and are shown by representative sera. Forty-six subjects had IAAs with the A8-10/13-dependent binding pattern represented in the top left panel. Six subjects had IAAs with the A8-10/13/B28-30-independent binding pattern represented in the middle left panel. Three subjects had IAA with the B30-dependent binding pattern represented in the top right panel. Three subjects had the B28/29-dependent binding pattern represented in the middle right panel. Seven subjects had the B28-30-dependent binding pattern represented in the bottom panel. (C) Competitive inhibition of IAA binding to Tyr ${ }^{14} \mathrm{~A}\left[{ }^{125} \mid\right.$ ] human insulin using human proinsulin (filled circles) for each of the sera shown in B. The dotted line represents competition with human insulin. 
Table 1

Characteristics of IAA and autoantibody development in IAA-positive relatives

\begin{tabular}{|c|c|c|c|c|c|c|c|}
\hline Case no. & Affinity ${ }^{A}$ & $\begin{array}{l}\text { A8-10/13 } \\
\text { dependent }\end{array}$ & $\begin{array}{c}\text { B28/29 } \\
\text { dependent }\end{array}$ & $\begin{array}{c}\text { B30 } \\
\text { dependent }\end{array}$ & $\begin{array}{c}\text { Proinsulin } \\
\text { binding }\end{array}$ & $\begin{array}{l}\text { Autoantibody } \\
\text { on follow-up }\end{array}$ & $\begin{array}{c}\text { HLA DR4- } \\
\text { positive }\end{array}$ \\
\hline $1-42$ & High & $\underline{\text { Yes }}$ & No & $\underline{\text { No }}$ & $\underline{\text { Yes }}$ & Multiple & $32 / 41^{F}(78 \%)$ \\
\hline 43,44 & $\underline{\text { High }}$ & $\underline{\text { Yes }}$ & $\underline{\text { No }}$ & $\underline{\text { No }}$ & $\underline{\text { Yes }}$ & Single & $02 / 4 \Gamma(100)$ \\
\hline 45,46 & Low & $\underline{\text { Yes }}$ & $\underline{\text { No }}$ & $\underline{\text { No }}$ & $\underline{\text { Yes }}$ & Transient IAAs & \\
\hline $47^{B}$ & Low & $\overline{N o}$ & $\underline{\text { No }}$ & $\underline{\text { No }}$ & $\underline{\text { Yes }}$ & Multiple & $3 / 8(37 \%)$ \\
\hline $48-52$ & Low & No & $\underline{\overline{N o}}$ & $\underline{\text { No }}$ & $\overline{\text { Yes }}$ & Single & \\
\hline 53,54 & Low & No & $\overline{Y e s}$ & $\underline{\text { No }}$ & $\overline{\mathrm{No}}$ & Single & \\
\hline 55 & Low & No & Yes & $\underline{\text { No }}$ & No & Transient IAAs & \\
\hline$(42 b)^{c}$ & Low & $?^{D}$ & No & $\overline{Y e s}$ & Yes & Multiple & \\
\hline 56,57 & Low & $?$ & $\underline{\text { No }}$ & Yes & No & Single & $5 / 13(38 \%)$ \\
\hline 58 & Low & $?$ & $\underline{\text { No }}$ & Yes & No & Transient IAAs & \\
\hline 59 & Low & $?$ & $\overline{\text { Yes }}$ & Yes & No & MultipleE & \\
\hline $60-65$ & Low & $?$ & Yes & Yes & No & Single & \\
\hline
\end{tabular}

AHigh affinity is greater than $10^{9} \mathrm{I} / \mathrm{mol}$. ${ }^{\mathrm{B}} \mathrm{Child}$ that developed high-affinity IAAs and multiple islet autoantibodies on follow-up. CLow-affinity IAA component in child with mixed IAA populations. DUnable to determine whether residue changes in A8-10 or A13 affect binding. EOnly child that had GAD antibodies prior to developing IAAs. FHLA typing was not performed in 4 subjects. Underlining indicates characteristics associated with multiple islet autoantibodies and diabetes.

IAA epitope and affinity are related to proinsulin binding. The proinsulin molecule includes a connecting peptide that alters the conformation of $\mathrm{COOH}$-terminal residues of the insulin $\mathrm{B}$ chain (10). We therefore asked whether binding to proinsulin could distinguish affinity- and epitope-related IAA reactivity (Figure 7C and Table 1). All B28-B30-independent IAAs, including all high-affinity IAAs, were completely inhibited by both insulin and proinsulin. In marked contrast, all but 1 of the B28-B30-dependent IAAs were poorly inhibited with proinsulin $(P<0.0001)$. The one exception (case $42 \mathrm{~b}$ in Table 1 ) is striking, since this is the low-affinity IAA component in the child with mixed IAA populations. The low-affinity IAAs in this child were uninhibited with porcine insulin and sheep A8his insulin, both of which have residue changes at position B30, but, unlike all other B30-dependent IAAs, were readily inhibited with human proinsulin. The highaffinity IAA component in this child had A8-13-dependent proinsulin binding. Also of note is that the low-affinity IAAs in the first positive sample of the child who subsequently developed multiple islet autoantibodies (case 47) were inhibited with proinsulin. Finally, 2 children had IAAs with affinity less than $10^{9} \mathrm{l} / \mathrm{mol}$ with A8-13-dependent and proinsulin binding, and in both cases IAAs were transient (cases 45 and 46).

\section{Discussion}

The affinity of IAAs was found to vary considerably among IAApositive relatives. Although IAAs within individual subjects appeared relatively homogeneous, they ranged from very highaffinity IgG in most individuals to low-affinity, cold-reactive, IgM antibodies in others. High-affinity IAA responses were most frequent when IAAs developed at a very young age and in relatives who had the $D R B 1 * 04$ allele. IAAs were of high affinity already in the first IAA-positive sample in almost all IAA-positive relatives who developed multiple islet autoantibodies and all who developed T1DM. In contrast, relatives who remained positive only for IAAs or became islet autoantibody-negative usually had loweraffinity IAAs that did not increase in affinity on follow-up. Highaffinity IAAs differed from lower-affinity IAAs in their insulin binding characteristics in a manner consistent with distinct epitope recognition and, in contrast to the lower-affinity IAAs, always bound strongly to proinsulin. The findings indicate that the nature of early exposure to (pro)insulin is relevant to disease pathogenesis, and that IAA affinity and epitope reactivity can classify stages of autoimmunity against islet cells and stratify diabetes risk.

This is the first study to examine islet autoantibody affinity over time and, in particular, from a very young age close to the development of islet autoimmunity. The affinity of IAAs was relatively fixed from the time of first detection and was already very high in children who were IAA positive at age 9 months, indicating that the IAA response can mature quickly. Many of these children were IAA negative at birth, indicating that the high-affinity IAAs observed at age 9 months represents a mature response in the child and not residual maternal insulin antibodies in most cases. Only one child who was IAA positive at 9 months had clear evidence of an evolving response, evidenced by the coexistence of high- and low-affinity IAAs at age 9 months and progressive replacement of the low-affinity IAAs by highaffinity IAAs. A limitation of the study is that we have focused on IAA-positive relatives of patients with T1DM, and although 2 IAA-positive nondiabetic control subjects had low-affinity IAAs, the findings of our study may not be true for IAA-positive subjects who do not have a family history of T1DM.

Two other studies have examined IAA affinity $(11,12)$. Unlike this study, which used a protein A-based radiobinding assay, the 2 previous studies used a polyethylene glycol-based radiobinding assay to determine affinity. Both studies reported IAAs of high affinity, and one reported that IAAs within the same subject includes both highand very low-affinity antibodies. The high-affinity IAAs reported in both studies have $K_{d}$ values similar to what we have found in the majority of relatives. We did not, however, find clear evidence for the coexistence of low-affinity IAAs, except in 1 subject. Binding curves for most sera tested were consistent with a 1-site binding model and suggest that there is 1 predominant IAA population within individual subjects. We cannot, however, exclude the presence of a small component of lower-affinity IAAs that is undetectable by the analytical system used. The finding that patients with T1DM produce high-affinity antibodies against exogenously administered insulin is consistent with previous reports (12-14). Other studies have shown that naturally occurring polyreactive autoantibodies can bind insulin with low affinity (15). Two relatives from our cohort had hightiter IgM cold-reactive low-affinity IAAs. These were not the same as the polyreactive antibodies previously described, since, unlike the polyreactive antibodies, they could not be inhibited by excess tetanus toxoid or single-stranded DNA (data not shown). IAAs with distinct binding characteristics (16), proinsulin autoantibodies (17), and high-titer, low-affinity IAAs in subjects with the rare insulin autoimmune hypoglycemia $(18,19)$ have also been described. 
The findings of this study are relevant to both pathogenesis and prediction of T1DM. With respect to pathogenesis, they indicate that the encounter between antigen and the immune system that gives rise to diabetes-relevant IAAs is likely to be of sufficient duration to evoke a high-affinity IgG response. IAAs that were of affinity less than $10^{9} \mathrm{l} / \mathrm{mol}$ rarely led to multiple islet autoantibodies, even when their epitope reactivity was the same as that of the diabetes-relevant IAAs. The findings also indicate that there are probably distinct interactions between the immune system and antigen that evoke IAAs that are of lower affinity or predominantly $\operatorname{IgM}$, and that these events are of insufficient magnitude or quality to start the cascade of multiple islet autoantibody responses that are associated with the development of T1DM. These low-affinity responses appear more likely to occur in the absence of the HLA DRB ${ }^{*} 04$ allele and in children over the age of 2 years. Moreover, some of these lower-affinity IAA responses became seronegative, as might be expected if the immunizing event was relatively brief and of low intensity.

The very early appearance of high-affinity IAAs with uniform binding characteristics in almost all children who subsequently developed multiple autoantibodies or diabetes suggests a consistent mode of immunization. The binding characteristics of the high-affinity diabetes-relevant IAAs were very similar to those previously described (11) and indicated that autoantibody binding is affected by changes in regions $\mathrm{A} 8-\mathrm{A} 13$ and unaffected by changes at the $\mathrm{COOH}$-terminal end of the $\mathrm{B}$ chain. These autoantibodies also bound to proinsulin with high affinity. In contrast, the majority of the IAAs seen in children who did not progress to multiple islet autoantibodies bound poorly to insulin molecules altered in the $\mathrm{B} 28-30$ residues, and to proinsulin. It is conceivable, therefore, that the early immunizing event that precipitates the autoimmunity leading to T1DM involves the exposure of proinsulin to the immune system, and that exposure to proinsulin preferentially results in IAAs with A8-13-dependent binding. From a viewpoint of structure, both the $\mathrm{A} 8-13$ and the $\mathrm{B} 29-30$ sites are accessible on the surface of monomeric and hexameric insulin $(20,21)$, whereas residues $\mathrm{B} 29$ and $\mathrm{B} 30$ are conformationally altered in proinsulin because of covalent bonding between B30 and A1 (10). Also consistent with a proinsulin immunization model, autoreactive T cells against proinsulin-specific peptides have been detected in patients with T1DM or preclinical T1DM and in HLA DR4 transgenic mice (22-26). Proinsulin-specific antibodies, however, were rarely found in our cohort and were never found prior to the appearance of IAAs (27). Moreover, insulin antibodies generated after insulin treatment in patients with T1DM, type 2 diabetes, or gestational diabetes were similar to the high-affinity IAAs (A13 dependent, proinsulin reactive), suggesting that exposure to proinsulin is not essential in order to generate diabetes-relevant IAAs (K. Koczwara et al., unpublished observations). Regardless of whether exposure to proinsulin or insulin leads to diabetes-relevant autoimmunity, it is likely that some of the lower-affinity IAAs result from immunization to a molecule(s) other than insulin or proinsulin. Persistence of the response without transition to high-affinity IAAs is likely to reflect either prolonged presence of antigen that is not insulin (IAAs resulting from cross-reactivity) or continuous exposure to insulin that does not become limiting for clonal antigen competition. The rare occurrence of transition from low- to highaffinity IAAs indicates that the presence of B lymphocytes that have low-affinity receptors for insulin is unlikely to directly lead to higher-affinity IAAs through antigen presentation or affinity maturation. Exceptions to this were observed in 2 children who had low-affinity IAAs that were proinsulin reactive.

With respect to prediction, the findings indicate that the measurement of IAA affinity in children who are only IAA-positive will be helpful in distinguishing who is more likely to develop multiple islet autoantibodies and to progress to T1DM. Identification of the low-affinity IAAs could be achieved by competitive binding with a few critical concentrations of cold insulin, insulin analog, or proinsulin, or, for some sera, simply by measurement of IAAs at room temperature or against alternatively labeled insulin. In view of the relatively high frequency of IAAs that is detected in young children, these modifications to the IAA assay will prove useful for improving specificity.

\section{Methods}

Study cohort. BABYDIAB prospectively follows offspring of parents with T1DM from birth with venous blood sampling and collection of questionnaire data at birth (cord blood) and at ages 9 months and 2, 5, 8, and 11 years (8). A total of 1,610 children were recruited at birth and followed at least until the 9-month visit. The median follow-up time was 6.5 years, range 9 months to 12.5 years, for a total of 9,480 subject years. Sera from all participants of the BABYDIAB study were tested for the presence of IAA, GAD antibodies, and IA-2 antibodies at follow-up visits, and frequent blood samples (3- to 12-month intervals) were subsequently obtained from antibody-positive children. All children were prospectively monitored for the development of diabetes, which was diagnosed using oral glucose tolerance tests by WHO/American Diabetes Association criteria (28). Monitoring in autoantibody-positive children was performed by oral glucose tolerance tests every 6-12 months and measurement of monthly random blood glucose values. Written informed consent was obtained from the parents. The study was approved by the ethical committee of Bavaria, Germany (Bayerische Landesärztekammer no. 95357).

Subjects and samples. A total of 68 BABYDIAB children developed IAAs that were confirmed positive in a consecutive $(n=66)$ or subsequent follow-up sample. Fifty-six of the 68 children were selected for our study on the basis of having sufficient serum available from their first positive sample for IAA affinity measurement. Of these 56 children, 16 were IAA positive at age 9 months, a further 26 became IAA positive at age 2 years, and another 14 became IAA positive at age 5 or 8 years. Twenty-three also had antibodies to GAD or IA-2 in their first antibody-positive sample (1 had GAD antibodies prior to IAAs), another 15 developed GAD and/or IA- 2 antibodies in follow-up samples, and 18 either remained IAA positive only $(n=13)$ or became islet autoantibody negative $(n=5)$ on follow-up. Twenty of the 56 children developed diabetes (median follow-up after first autoantibodypositive sample for all 56 children, 3.3 years; IQR, 1.9-5.2 years).

In order to verify the findings from the BABYDIAB cohort, IAA affinity was measured in a second group of 16 IAA-positive nondiabetic relatives selected from the Munich family study cohort (median age, 9.1 years; IQR, 6.0-15.1 years) (29). Eleven of these 16 relatives developed multiple islet autoantibodies, and 8 developed T1DM.

IAA affinity was also determined in sera from 11 patients with T1DM (median age, 13.1 years; IQR, 11.6-16.3 years) who had been treated with insulin injections for a median duration of 3.1 years (IQR, 2.8-3.3 years). All patients had high-titer insulin antibodies (median IAA titer, 70.8 units; IQR, 42.2-184.2 units) consistent with the presence of exogenously induced insulin antibodies.

Islet autoantibody measurements. IAAs, GAD antibodies, and IA-2 antibodies were measured by protein $\mathrm{A} / \mathrm{G}$ radiobinding assays as previously described $(2,30)$ using $T y r^{14} \mathrm{~A}\left[{ }^{125} \mathrm{I}\right]$-labeled human insulin (Aventis Pharma Deutschland GmbH, Bad Soden, Germany) and $\left[{ }^{35} \mathrm{~S}\right]$ methionine-labeled 
in vitro-translated recombinant human GAD65 and IA-2, respectively. For each antibody, results were expressed as arbitrary units that were derived from a standard curve. For IAAs, there was a linear relationship between the units and the immunoprecipitated counts per minute (cpm) that extended beyond 800 units (around 5,000 cpm). No samples had IAAs above 800 units. The thresholds for positivity in each assay corresponded to the 99th percentile of control subjects. These assays had sensitivities (positivity in 50 patients with new-onset T1DM) and specificities (negativity in 100 blood donors) of $84 \%$ and $96 \%$, respectively, for GAD antibodies; $66 \%$ and $100 \%$, respectively, for IA-2 antibodies; and $64 \%$ and $99 \%$, respectively, for IAAs in the Third Diabetes Autoantibodies Standardization Proficiency workshop. For some experiments, $\left[{ }^{125} \mathrm{I}\right]$ insulin labeled at amino acid 19 of the insulin A chain $\left(\operatorname{Tyr}^{19} \mathrm{~A}\right.$; Aventis Pharma Deutschland $\mathrm{GmbH}$; kindly provided by Raymond Oekonomopulos) was used to determine IAA binding.

IgG subclasses and isotypes of IAAs were determined by radiobinding assays as previously described (31) using IgG subclass- or isotype-specific biotin-labeled mouse anti-human mAb's (BD, San Diego, California, USA) bound on Sepharose 4B streptavidin beads (Zymed Laboratories Inc., South San Francisco, California, USA). The antibodies used were mouse mAb's against human IgG1 (clone G17-1), IgG2 (clone G18-21), IgG3 (clone HP6047; Zymed Laboratories Inc.), IgG4 (clone JDC-14), and IgM (clone G20-127). Nonspecific binding was determined for each serum using beads coated with anti-rat IgM mAb (clone G53-238). Results for IAA subclasses were expressed as nanounits insulin bound per milliliter after subtraction of binding with the anti-rat IgM-coated beads. The cutoff for positivity for each IAA IgG subclass and isotypes was $150 \mathrm{nU} / \mathrm{ml}$ (mean plus 3 SD of IAA-negative control subjects).

Cytoplasmic islet cell autoantibodies were detected by the indirect immunofluorescence test on unfixed cryostat sections of human pancreas from an organ donor with blood group 0 as previously described (2).

IAA affinity measurement. Affinity was measured by competitive binding experiments. Briefly, $5 \mu \mathrm{l}$ serum was incubated in duplicate for 72 hours in TBT buffer (50 mM Tris, $1 \%$ Tween-20, pH 8.0) in the presence of 7.85 femtomoles of human ${ }^{[125 \mathrm{I}]}$ insulin (Aventis Pharma Deutschland $\mathrm{GmbH}$ ) labeled at the tyrosine at position 14 of the A chain of the insulin molecule $\left(\mathrm{Tyr}^{14} \mathrm{~A}\right)(10 \mu \mathrm{Ci} / \mathrm{ml} ; 0.143 \mathrm{nM})$ with or without increasing quantities of unlabeled human insulin $\left(2.6 \times 10^{-17}\right.$ to $1.7 \times 10^{-8} \mathrm{~mol}$; Aventis Pharma Deutschland $\mathrm{GmbH}$ ) in a final volume of $55 \mu \mathrm{l}$. Immune complexes were precipitated using protein $\mathrm{A} / \mathrm{G}$-Sepharose, and washed as previously described for the IAA assay (30). Bound $\left[{ }^{125} \mathrm{I}\right]$ insulin was measured using a gamma counter (Packard Instrument Co., Meriden, Connecticut, USA). Nonspecific binding determined as the binding of an IAA-negative control serum to $\left[{ }^{125} \mathrm{I}\right]$ insulin in presence of $1.38 \mathrm{nmol}$ of unlabeled human insulin was subtracted for each experiment, and results were expressed as counts per minute (cpm). $\mathrm{IC}_{50}$ and $K_{\mathrm{d}}$ values were calculated using the GraphPad Prism 3 program (GraphPad Software Inc., San Diego, California, USA), and IAA affinity was expressed as reciprocal $K_{\mathrm{d}}$ values $(1 / \mathrm{mol})$. The reproducibility of IAA affinity measurements was determined from replicates of an IAA-positive serum that was included in each experiment. The mean + SD affinity of this sample was $1.69 \times 10^{11}+0.28 \times 10^{11} \mathrm{l} / \mathrm{mol}$ (interassay coefficient of variation, $16.2 \% ; n=10$ experiments). In some experiments, $\left[{ }^{125} \mathrm{I}\right]$ insulin labeled at position 19 of the A chain $\left(\mathrm{Tyr}^{19} \mathrm{~A}\right.$; Aventis Pharma
Deutschland $\mathrm{GmbH})$ or at position 16 of the $\mathrm{B}$ chain of insulin $\left(\mathrm{Tyr}^{16} \mathrm{~B}\right.$; Aventis Pharma Deutschland GmbH; kindly provided by Raymond Oekonomopulos) was used instead of $\mathrm{Tyr}^{14} \mathrm{~A}\left[{ }^{125} \mathrm{I}\right]$ insulin.

Epitope analysis. In order to determine whether IAAs from different subjects bound similar epitopes, binding to $\mathrm{Tyr}^{14} \mathrm{~A}\left[{ }^{125} \mathrm{I}\right]$ insulin was competed with increasing amounts of cold insulin from different species (human [Insuman Rapid; Aventis Pharma Deutschland GmbH], porcine [Aventis Pharma Deutschland GmbH], and salmon [coho salmon, Oncorbynchus kisutch; a gift from Erika M. Plisetskaya, University of Washington, Seattle, Washington, USA]), with insulin analogs (human B28lysB29pro [Humalog; Eli Lilly and Co., Indianapolis, Indiana, USA], human A13trpB28lysB29pro [a gift from Panayotis G. Katsoyannis, Mount Sinai School of Medicine, New York University, New York, New York, USA], and sheep A8his [from P.G. Katsoyannis]), and with human proinsulin (Eli Lilly and Co.). The competing antigens were added to the standard IAA radiobinding assay at increasing concentrations ranging from $1.7 \times 10^{-10} \mathrm{M}$ to $6.8 \times 10^{-6} \mathrm{M}$.

HLA typing. HLA DRB1/DQB1 alleles were typed using PCR-amplified genomic DNA and sequence-specific oligonucleotide probes (32).

Statistical analysis. The Mann-Whitney $U$ test or the Kruskal-Wallis $H$ test was used to compare IAA affinities between groups. Multivariate analysis for significant variables was performed by multiple regression. Fisher's exact test was used to compare prevalence between groups. Spearman's correlation was used to determine the correlation between variables. Life table analysis was used to compare outcome (multiple-autoantibody status and diabetes status) for children with different IAA affinity categories $\left(<10^{9}\right.$ $1 / \mathrm{mol}$ or $>10^{9} \mathrm{l} / \mathrm{mol}$ ). The time between first IAA-positive sample and first multiple islet autoantibody-positive sample or last sample was defined as the time to event for multiple-autoantibody status. The time between first antibody-positive sample and diagnosis of diabetes or last contact was defined as the time to event for diabetes status. Significance of differences between groups was determined using the log rank test. For all analyses, a 2-tailed $P$ value of 0.05 was considered significant. Statistical analyses were performed using the Statistical Package for Social Science (SPSS 11.0; SPSS Inc., Chicago, Illinois, USA).

\section{Acknowledgments}

This work was supported by grants from Deutsche Forschungsgemeinschaft (ZI 310/12-5), the Juvenile Diabetes Research Foundation (JDRF no. 1-2003-646), and the Eli Lilly Research Foundation. The authors thank Raymond Oekonomopulos for providing radiolabeled insulin, Panayotis G. Katsoyannis for providing insulin analogs, Erika M. Plisetskaya for providing fish insulin, Ulrike Mollenhauer and Karolina von Dalwigk for technical support, and Markus Walter and Michael Hummel for clinical assistance.

Received for publication February 11, 2004, and accepted in revised form June 22, 2004.

Address correspondence to: Ezio Bonifacio, Diabetes Research Institute, Kölner Platz 1, 80804 Munich, Germany. Phone: 49-89-3079-3121; Fax: 49-89-3081-733; E-mail: Ezio.Bonifacio@ lrz.uni-muenchen.de.
1. Atkinson, M.A., and Eisenbarth, G.S. 2001. Type 1 diabetes: new perspectives on disease pathogenesis and treatment. Lancet. 358:221-229.

2. Ziegler, A.G., Hummel, M., Schenker, M., and Bonifacio, E. 1999. Autoantibody appearance and risk for development of childhood diabetes in offspring of parents with type 1 diabetes: the 2-year analysis of the German BABYDIAB Study. Diabetes. 48:460-468.

3. Kimpimaki, T., et al. 2002. Natural history of beta-cell autoimmunity in young children with increased genetic susceptibility to type 1 diabetes recruited from the general population. J. Clin. Endocrinol. Metab. 87:4572-4579.

4. Colman, P.G., et al. 2000. Islet autoimmunity in infants with a Type I diabetic relative is common but is frequently restricted to one autoantibody. Diabetologia. 43:203-209.

5. Yu, L., et al. 2000. Early expression of antiinsulin autoantibodies of humans and the NOD mouse: evidence for early determination of subsequent diabetes. Proc. Natl. Acad. Sci. U. S. A. 97:1701-1706.

6. Burnet, F.M. 1961. The new approach to immunology. N. Engl. J. Med. 264:24-34.

7. Wabl, M., Cascalho, M., and Steinberg, C. 1999. Hypermutation in antibody affinity maturation. Curr. Opin. Immunol. 11:186-189.

8. Hummel, M., et al. 2004. Early appearance of islet 
autoantibodies predict childhood type 1 diabetes in offspring of diabetic parents. Ann. Intern. Med. 140:882-886.

9. Bingley, P.J., Bonifacio, E., and Mueller, P.W. 2003. Diabetes Antibody Standardization Program: first assay proficiency evaluation. Diabetes. 52:1128-1136

10. Snell, C.R., and Smyth, D.G. 1975. Proinsulin: a proposed three-dimensional structure. J. Biol. Chem. 250:6291-6295.

11. Castano, L., Ziegler, A.G., Ziegler, R., Shoelson, S., and Eisenbarth, G.S. 1993. Characterization of insulin autoantibodies in relatives of patients with type I diabetes. Diabetes. 42:1202-1209.

12. Brooks-Worrell, B.M., Nielson, D., and Palmer, J.P. 1999. Insulin autoantibodies and insulin antibodies have similar binding characteristics. Proc. Assoc. Am. Physicians. 111:92-96.

13. Vahasalo, P. 1992. Autoantibodies to insulin have similar affinity to that of antibodies to exogenous insulin but lower binding capacity. Eur. J. Clin. Invest. 22:772-776.

14. Baxter, R.C., Yue, D.K., and Turtle, J.R. 1976. Equilibrium binding studies of insulin antibodies in diabetic subjects. Clin. Chem. 22:1089-1094.

15. Casali, P., Nakamura, M., Ginsberg-Fellner, F., and Notkins, A.L. 1990. Frequency of B cells committed to the production of antibodies to insulin in newly diagnosed patients with insulin-dependent diabetes mellitus and generation of high affinity human monoclonal IgG to insulin. J. Immunol. 144:3741-3747.

16. Wilkin, T., Mirza, I., Armitage, M., Casey, C., and
Scott-Morgan, L. 1988. Insulin autoantibody polymorphisms with greater discrimination for diabetes in humans. Diabetologia. 31:670-674.

17. Kuglin, B., Gries, F.A., and Kolb, H. 1988. Evidence of IgG autoantibodies against human proinsulin in patients with IDDM before insulin treatment. Diabetes. 37:130-132.

18. Goldman, J., et al. 1979. Characterization of circulating insulin and proinsulin-binding antibodies in autoimmune hypoglycemia. J. Clin. Invest. 63:1050-1059.

19. Hirata, Y. 1983. Methimazole and insulin autoimmune syndrome with hypoglycemia. Lancet. 2:1037-1038

20. Adams, M.J., et al. 1969. Structure of rhombohedral 2-zinc insulin crystals. Nature. 224:491-495.

21. Smith, G.D., Swenson, D.C., Dodson, E.J., Dodson, G.G., and Reynolds, C.D. 1984. Structural stability in the 4-zinc human insulin hexamer. Proc. Natl. Acad. Sci. U. S. A. 81:7093-7097.

22. Arif, S., et al. 2004. Autoreactive T cell responses show proinflammatory polarization in diabetes but a regulatory phenotype in health. J. Clin. Invest. 113:451-463. doi:10.1172/JCI200419585.

23. Dubois-LaForgue, D., Carel, J.C., Bougneres, P.F., Guillet, J.G., and Boitard, C. 1999. T-cell response to proinsulin and insulin in type 1 and pretype 1 diabetes. J. Clin. Immunol. 19:127-134.

24. Durinovic-Bello, I., et al. 2004. Pro- and anti-inflammatory cytokine production by autoimmune $\mathrm{T}$ cells against preproinsulin in HLA-DRB1*04, DQ8 Type 1 diabetes. Diabetologia. 47:439-450.

25. Rudy, G., et al. 1995. Similar peptides from two beta cell autoantigens, proinsulin and glutamic acid decarboxylase, stimulate $\mathrm{T}$ cells of individuals at risk for insulin-dependent diabetes. Mol. Med. 1:625-633.

26. Congia, M., Patel, S., Cope, A.P., De Virgiliis, S., and Sonderstrup, G. 1998. T cell epitopes of insulin defined in HLA-DR4 transgenic mice are derived from preproinsulin and proinsulin. Proc. Natl. Acad. Sci. U. S. A. 95:3833-3838.

27. Hummel, M., et al. 2001. Proinsulin-specific autoantibodies are relatively infrequent in young offspring with pre-type 1 diabetes. Diabetes Care. 24:1843-1844.

28. American Diabetes Association. 1997. Report of the Expert Committee on the Diagnosis and Classification of Diabetes Mellitus. Diabetes Care. 20:1183-1197.

29. Achenbach, P., et al. 2004. Stratification of type 1 diabetes risk on the basis of islet autoantibody characteristics. Diabetes. 53:384-392.

30. Naserke, H.E., Bonifacio, E., and Ziegler, A.G. 1999. Immunoglobulin $G$ insulin autoantibodies in BABYDIAB offspring appear postnatally: sensitive early detection using a protein A/G-based radiobinding assay. J. Clin. Endocrinol. Metab. 84:1239-1243.

31. Bonifacio, E., Scirpoli, M., Kredel, K., Fuchtenbusch, M., and Ziegler, A.G. 1999. Early autoantibody responses in prediabetes are IgG1 dominated and suggest antigen-specific regulation. J. Immunol. 163:525-532.

32. Walter, M., et al. 2003. IDDM2/insulin VNTR modifies risk conferred by IDDM1/HLA for development of Type 1 diabetes and associated autoimmunity. Diabetologia. 46:712-720. 